\title{
$\mathfrak{I} \mathfrak{n} \mathfrak{a} \mathfrak{a} \mathfrak{t} \mathfrak{s}-\mathfrak{d} \mathfrak{e} \mathfrak{e} \mathfrak{z} \mathfrak{e} \mathfrak{d} \mathfrak{h} \mathfrak{i} \mathfrak{s}$.
}

\section{Die $\mathfrak{E} e$ hrerbildung.}

$\S 1$. Begenwärtige Brunblage für bie Refrerbilbung.

8 2. Boibemertung.

A. Aufnaljue in riu sentinar.

3. Rebenßalter und Borbilbung für bie $\mathfrak{A}$ ufnahmeprüfung.

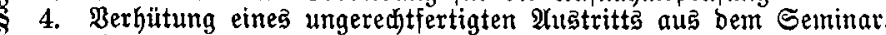

5. Mevers für ßräparanben.

6. Borbenterfung.

\section{B. đie L'eljererpriifuturu.}

7. \$3erbinbung ber \$rüfung ber Rehramts̄bewerber mit berjenigen ber Seminar= abiturienten.

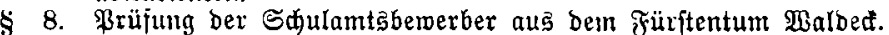

9. Beitbauler für bie Brüfung.

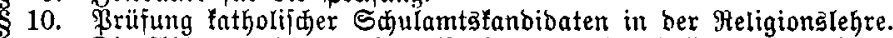

11. Die 2 blegung ber sweiten \$rüfung vor ber befinitiven Anjtellung.

\$12. Zweá ber zweiten Prüfung.

12.a Einribtung Der zlveiten \$rüfung.

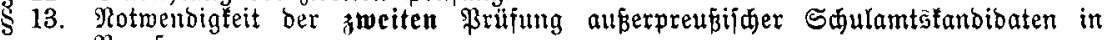
Breusen.

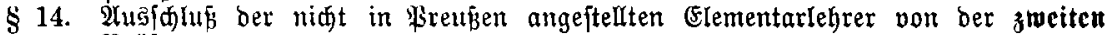
Prüfung.

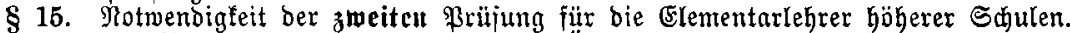

\$16. Bulaîung zux zweiten \$rüfung beim übertritt an Taubftummenanjalten. (1-2.)

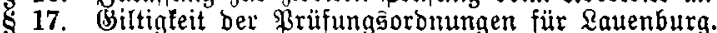

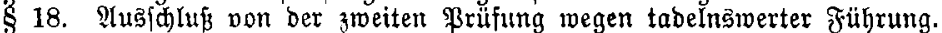

19. Infertigung (d) riftlider Arbeiten feitens ber \&ehrer.

20. Begriundung von Areis=2efyerbibliothefen.

\section{C. priifuttg für thliftelidullehrer.}

81. Mlgemeine Bemerfungen.

22. 2lnforderung in ber \$rüfung $2 c$.

23. Beredtigung ber \&ehrer, welhe früher pro schola et rectoratu geprüft worben fitto.

24. \$rüfungäfädur in ber \$rüfung für \&efrer an Mittelfăulen.

25. 3ulafiung ber אanbibaten ber Theologie unb ber \$hilofophie.

26. iłbergangābeftimmungen für ältere Lehrer.

27. Beredtigung ber ₹ehrer, welde in ben Jahren $1873 / 75$ im Seminar zu Bertin bie frembipradhlide Prüfung beftanden haber.

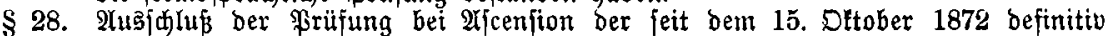
angefteltten Regrer.

\$29. 3ulajfung anfaltifder Staatäangeföriger zur Mittelfdullebrer= uno Reftorata prüfung zu Magbeburg.

$\$ 30$. Erlangung ber Befähigung für Mittelfdulen zc. burd) Beugnis einer wiffen[đaft liđen \$rüfungäommiffion.

§ 31. Termin zur Einziehung ber \$rüfungg̈gebühren.

\section{Hektoratsprïfung.}

\$32. $\ddot{\mathfrak{u} b e r}$ Reftoratşpriifung im allgemeinen.

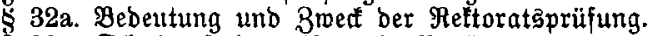

33. Sđulvoriteherprïfung in Berlin.

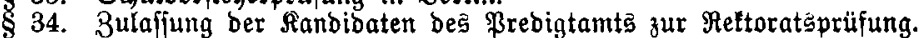

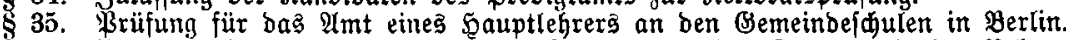

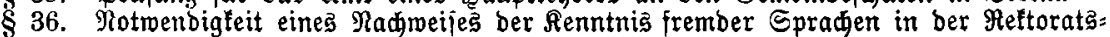
prüfung.

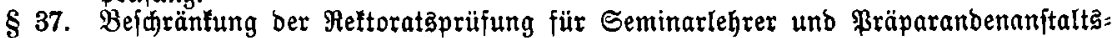
vorfteher. $(1-2$. 
§ 38. Zulaffung einesె vor bem 15. Dttober 1872 an einer Mittelidule angeftellten Lebrers zur Reftoratsprïfung.

839. 3ulaffurg ber Seminarlehrer zur ßeftoratsprüfung unter Dispenfation von ber TRittelfdullebrerprüfung.

\section{E. Aushilduny in der muftk und itn Turnen.}

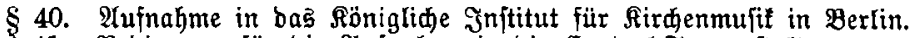

41. Bebingung für bie Alufnahme in bie Central=:urnantalt.

\$42. Feglement für bie \$rüfung der Turnlebrer.

\section{Finftellung.}

\$4. über bie 2 (njtellung im allgemeinen.

\$4. $\mathfrak{u}$ bertritt Der Seminarzöglinge in \$rivatverhältmiffe.

45. Bulafjung ber auङ bem Sentinar verwiejenen Zöglinge zum Shulbienft.

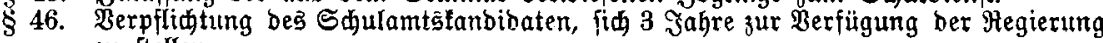
zu itellen.

47. Dreijäbrigeș Berbleiben in bem betreffenoen Regierunģ̧bez̧irf. (1-2.)

48. Wieberanftellung entlaffenter Relirer.

49. Proviforifhe unb befintitive Anftellung.

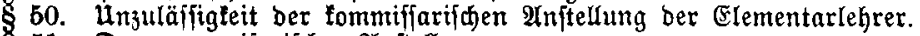

51. Dauer provijorifder Anjtellung.

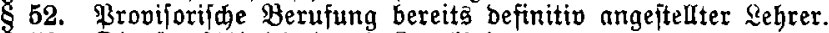

53. Dienjtunfähigteit burd Srantheit.

54. 2rnftĕlung fogenannter nidbt eraminierter Seminariften.

55. Beftimmungen über bas breijäbrige Berbleiben im Sdulamte in Branbenburg.

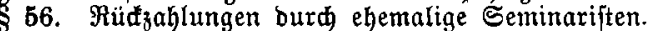

57. Rotwendigfeit ber 2 Ableiftung ber Militärpflidgt vor ber befinitiven Anftellung

58. Berufung burø einen \$ripatpatron.

59. Bofation für Rehrer an Privatpatronatştellen.

60. Bebeutung ber Bofation in $\mathfrak{B e z u g}$ auf Gehaltşeftietung.

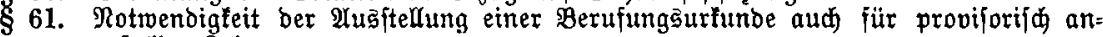
geftellen @efrer.

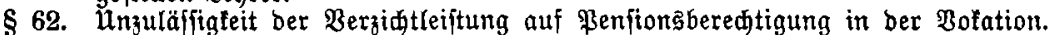

\$63. Stempelpflimtigfeit Der Botation. $(1-3$.

64. Form ber Entlaffung proviforifd angeftefler \&ehrer. (1-2.)

65. Bofation für Rehrer an ftäbtifden mehrflaffitgen Sdulen.

66. Berufung eines \&ehrers für eine beftimmte Slafie.

67. Berpflidutungent für Silfffenlehrer an mehrflaffigen Sd)ulen zur Ü̈bernahme von Sefritunden in anbern Flaffen.

\$68. Berufung für eine beftimmte Stelfe an Siönigliden 2Inftalten.

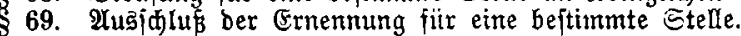

\$69a. Beitreije Bermaltung erlebigter Lehrerjtellen, bez̧w. Bertretung von Lehrern an benaぬbarten ङ઼ulen.

$\S 69$ b. Die Berufungăurfunbe, welde bei (Helegenheit ber Einfüfrung burd, ben Søul=

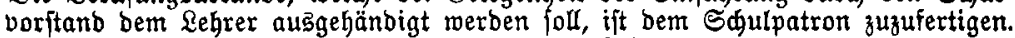

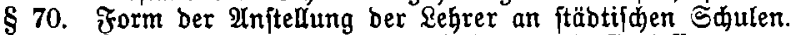

71. Sompetenz bezüglíb ber Unitellung uno Entlafjung.

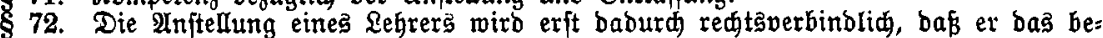
treffende Rehramt wirtlid antritt.

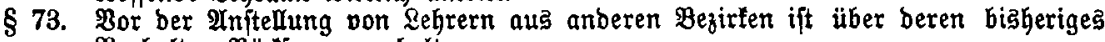
Berbalten Mütfrage su halten.

\section{Bejoldung der Rehrer.}

\$ 74. Benährleiftung eines fejten Einfommens burd ben Staat.

\section{A. Auskönmulide ferolàum!.}

\$ 75. Sorge ber Regierung für austömmlidae Befoloung.

75a. Bemeffung ber Befolbung eines Rehrers nad ben örtliden Bergältniffen.

76. Feftjęung unb erefutivifhe \$eitreibung von Behaltşzlagen.

77. Erhöhung ber Rehrerbefolbung.

78. Verpfliđtung jur Bewährung auşreiøenber Lehrerbefolbung.

79. Notrenbigleit und Buläffigteit fortidreitender Serbefierung. (1-2.)

80. Befriebigung ber Elementarfकulbebürfniffe feitens ber Stabtgemeinbe.

81. Berbefferung ber Befolbung in ben Stäbten. 
82. Đewährung einesิ †tanbes̄gemäßen Einfommeng für Ełementarlehrer.

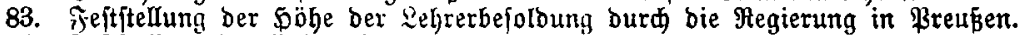

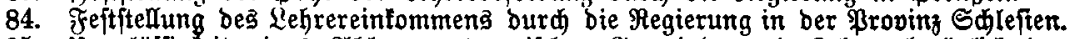

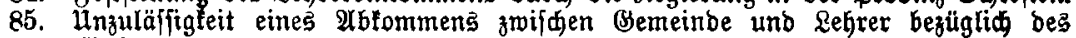
Einfommens.

86. Untechnung auḡwärtiger Dienftzeit bei ber Bemejiung ber Befoldurg.

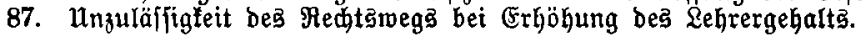

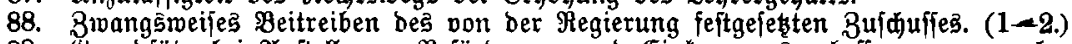

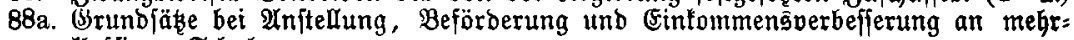
flajifigen Sdullen.

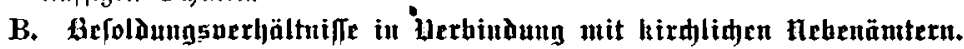

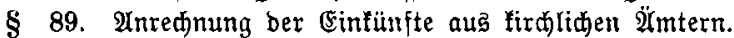

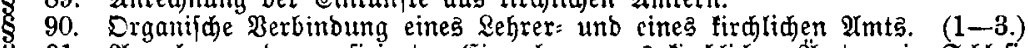

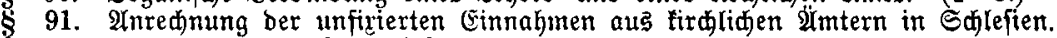

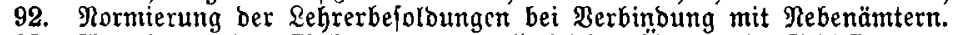

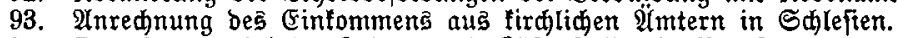

94. Dotation vereinigter \&ehrer= und Rüfterftellen in Breusen.

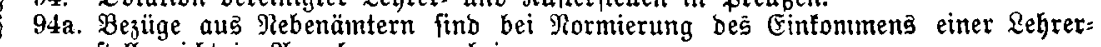
ftelle nidit in 2Inredinung zu bringen.

$\S$ 95. 2(ffđhaffung ber Sing: unb Bittumgänge ber Rehrer mit Sđultinbern.

\section{Hefoldung mährend der Baure des firizgshienftẹ.}

\$ 96. Dienfteinfommen ber Rommunalbeamten während ber Dauer bes Rrieģ̧oienfteă.

\$ 97. $\mathfrak{M}$ ufbringung ber Jioften für bie Bertretung währenb ber Arieg引zeit.

98. Dienfteinfommen ber \&ehrer während ifrer Stellung bei ber 2 rmee.

99. Fortbauer besె (Behaltşbezugeș für bie Beit ber Đilitiöroienjtleiftung. (1-2.)

100. Dotation einer Qehrerftelle bet geringer Sturbenzahl.

\section{Dicuftuoljunı und}

\$ 101. Rotwenbigfeit ber Bejdaffung von @ehrerwohnungen.

102. Bomftändigfeit von Sehrermohnungen.

103. Seizbarmachung von Rehrermohmungen.

104. Benutung ber Lehrerwobnung.

105. 2Arednung ber Dienftwohnung auf \&ehrereinfommen.

106. Bewährung freier $\mathfrak{B}$.

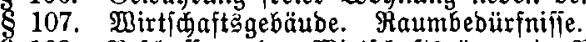

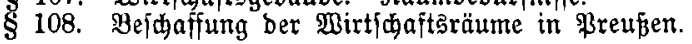

\section{E. Beputatholy.}

\$109. Brentholzquantum in ber \$rovinz \$reuken.

110. Dualität bes Deputatholzeș.

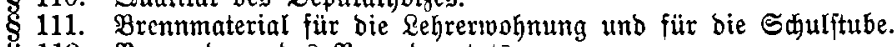

112. Berwentung des Brenndeputats.

113. Frhöhung bes Deputatä na屯 bauliđer Beränderung.

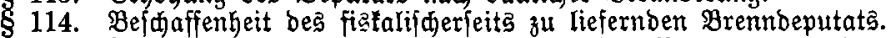

\$115. Bermenbung bes einent Refrer zuftehenden Brennholges. (1-2.)

116. Unterfagung einer eigenmäđtigen Beräuß̉erung in ber \$rovinz \$reuţen.

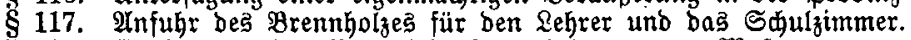

118. Ermittelung Des Brennbebarfs nad bem neuen $\mathfrak{M a}$.

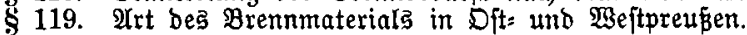

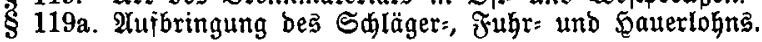

\section{F. Bas Sablland.}

§ 120. Beredtigung bes Rehrers zur Rukung bes Sdullanbe弓.

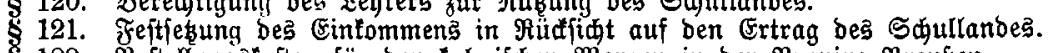

\$122. Beftellungśtoiten für ben fulmifđen Rorgen in ber \$roving \$reusen.

8123. Berpflidtung zur Beftellung be马 Sdulmorgens.

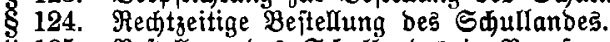

125. Beitellung bes Shullanbes in \$reuben. (1-5.)

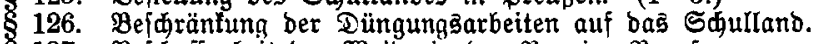

127. Befdaffenheit Der Beibe in ber \$roving \$reupen.

$\S 128$. Anlegung befonberer Sđulbrunnen.

G. Altergzulagen. (Uufiteigenbe Rehrergehälter.)

\$129. Fompetenzyerbältnifje bet Bewilligungen von 3 Llagen.

$\$ 130$. Svjtem ber थllterşzlagen. 


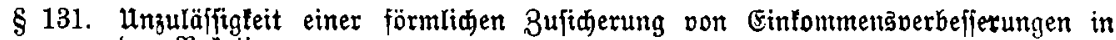
ben Botationen.

132. Ânredinung ber im öffentliden Sđulamte zugebradten Zeit.

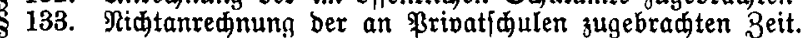

134. 2Inredinung ber Beit proviforifđer Anftellung in Bezug auf bie Dienitalterszulagen.

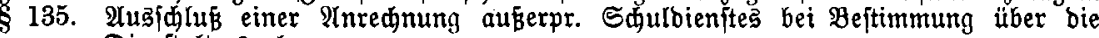
Dienftalterszzulage.

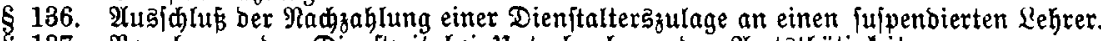

137. Beredinung ber Dienftzeit bei unterbredung ber 2 mtathätigfeit.

138. Nidtantedmung ber an einer \$rivatidule zugebradten Zeit.

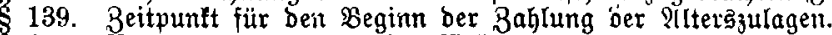

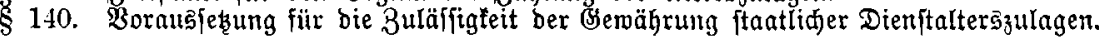

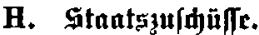

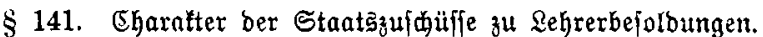

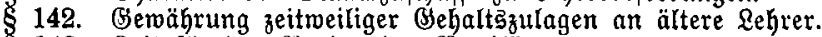

143. Beit für ben Beginn ber Bewilligung $2 c$.

144. Beranlaffung zur Entziehung ber Dientalterszulage.

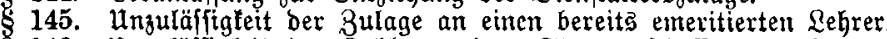

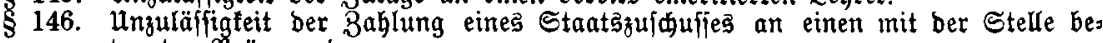
trauten \$räparanben.

\$ 147. ßezüge eines \$räparanden bei ber ßermaltung einer \&ehreritelle.

148. Remuneration für einen Präparanden.

\section{J. 3nhlungstermin fïr Prljrergebälter.}

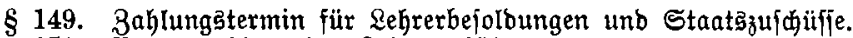

150. Borauşăhlung ber Sehrergefälter.

151. Zahlungätermin für interimifitifä angeftellte \&ehrer.

152. Bahlungatermin für 3ulagen ber Rehrer.

153. Termin für Zahlung von Refrerbefoldungäzulagen aus Staatsfonos.

\$ 154. Termin für Bahlung von Dienftalterz̧zlagen. Wegfall beim Tobe, ber Ėmeri= tierung ober Nerfekung bes Empfängers.

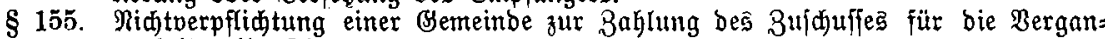
genfeit. $(1-2$.

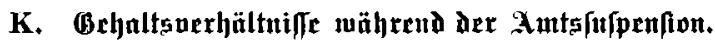

§ 156. Eingiehung bes rüaftänbigen BSehalts eines vom 2 mte fuppenbiert gewefenen Qehrerä itm Beriwaltungäwege.

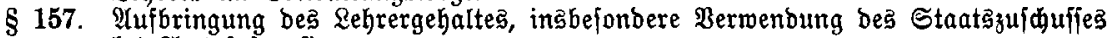
bei $\mathfrak{A} m+\mathfrak{s}\{$ ujpenfion.

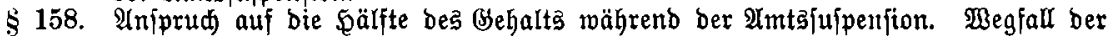
3ulagen aus Staatsfonos.

8 159. Gehalt引̧ahlung an Fufpenbierte Rehrer während ber Entfernung vom bisherigen $\mathfrak{A m t s f i t e .}$.

§ 160. Dişziplinarifde Huffidt. (Gehaltaz̧ahlung während ber Entfernung vom bişs Gerigen $\mathfrak{A m} \mathfrak{m}$ jit.

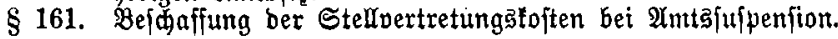

\section{Zarrídiedenxs.}

\$ 162. Shorniteinfegerlohn in Rehrermohnungen.

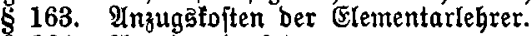

164. 2Auseinamber

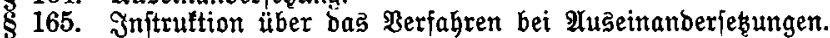

166. Auseinanberjebung im Beltungsbereid ber Sđuloronung vom 11. Dezember 1845.

167. Nułung ber Dbftbäume.

168. Nhłung ber Brunbftüffe.

169. Remuneration für Bertreturn einer vafanten Rehrerftelle.

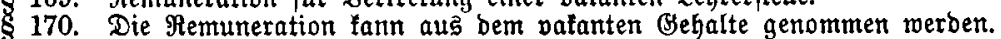

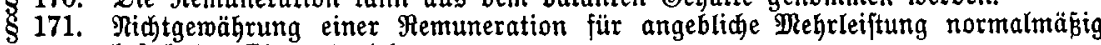
befoldeter Elementarlehrer.

M. (B)tudenżit für Ginterblicheur.

\$ 172. 2Ulerbödjite Rabineţorbre vom 27. 2pril 1816.

173. Gnabenfompetenz ber Sinterbliebenen.

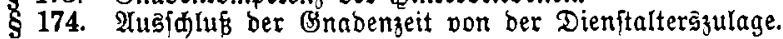


N. fjeiz̧uแn und Reininung der Gabulfulbe.

\$ 175. Entj̧äbigung für bie Beheizung. (1- -3.)

\$176. Berpflidbtung zur Meinigung unb 5eizutg bes Sdjulzimmers; Geranjiehung ber Sdulfinder.

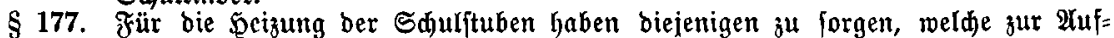
bringung ber Sdulunterhaltungstoften verpflidtet finb.

$\$$ 178. Beforgung bes Seizens ber Sdullofale.

179. Unnahme eines bejonbern நeizers.

$\$ 180$. Łeizungäanlayen.

\section{IV. (5nteritierung.}

\$181. Gefeł, betreffent bie \$enfionierung ber Befgrer und \&efrerinten v. 6. Juli 1885.

182. Berfahren bet unfreimilliger (5meritierung.

\$183. Enticheibung über ßenfionierung.

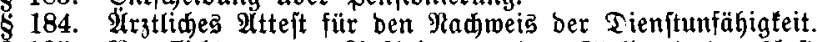

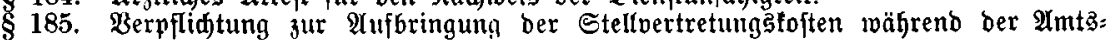
jupenfion uno bes Emeritengehalts.

$\$ 186$. Befiglagnahme von \$ejoldungen und \$enfionen.

\section{Beriönlide Berhältuiffe des $\mathfrak{E e h r e r s . ~}$}

\section{A. Allitïrüurrbültuiđe.}

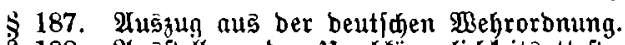

188. QHagfellung ber Unabtömmlideteitsattefte.

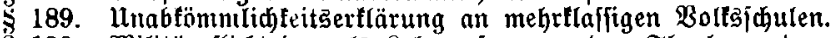

\$ 190. Mifitärpflidt ber als Lebrer fung erenden Theologen ber Brubersunität.

\$ 191. Begriff.

B. Mehenämter und Mebenhefinäftigutugn.

192. Frteilung von \$rivatunterridt. $(1-2$.

193. 3ur Erteilung von \$rivatunterridgt ift bie Erlaubnis ber Bebörbe nötig.

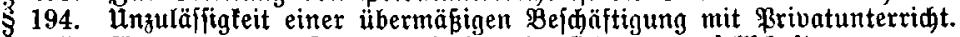

195. Agenturen von Feuer: und Sagelverfiderung gejellidaiten.

196. Bewerbebetrieb jeiten? ber Qehrer unb ber Angehörigen berjelben.

197. Лrompetenz bei Seftattung ber übernabme von Rebenämtern.

198. Rebenverbienft für \$rivatunterridtt und \&efreteinfomment.

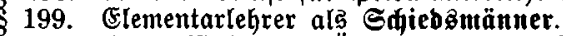

200. Unzuläffigfeit der übertragung bes Sđuulzewamtes an einen Qehrer.

201. Unterfagung ärjtlidjer Braria burfh bie Yefrer.

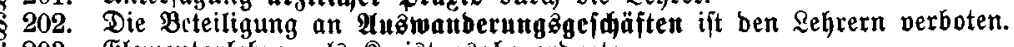

203. Elementarlehrer als Rreistagsabgeoronete.

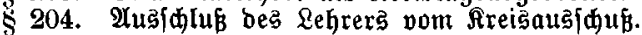

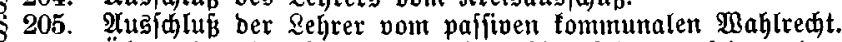

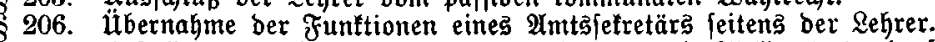

207. Beftellung von Sehrern als Standesbeamte unb Stellvertreter berfelber.

208. $\mathfrak{B a h l}$ bes Rehrers in ben Gemeindefirdenrat.

\$ 209. Berbot ber 2lüübung ber Эagd. $(1-3)$.

\section{Zisł̧ipliunturtfahren gegen Elrutrutarleljer.}

$\$ 210$. Befebs, betreffend bie Dienftuergehen ber nidt ridterlidjen Beamiten.

211. Bebingungen für Einteitung bes Dis̄ziplinarberfahrens gegen \&ehrer $2 c$.

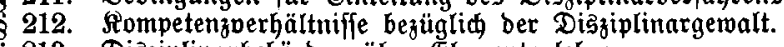

213. Dişziplinarbehörden über Ėlementarlehrer.

214. Berfabren bei Ent|đeibung in Disziplinarjađen wiber nidht ridjterlide Beamte.

215. Einftellung bes Disziplinarverfahrens gegen Qehrer im Raufe ber $\mathfrak{u}$ nterfudung.

216. Berfahren, nafbem ber 9 ngefduldoigte außer Berfolgung gejest morben ift.

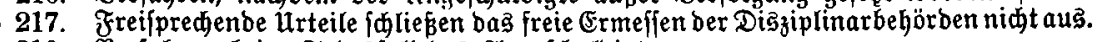

218. Merfahren beim Tobesfall bes Ingefdulbigten.

219. Berfabren bei Disziplinarunterjuffungen in 5annover.

220. Berufunggffrift in Disziplinarunterfudungâjąen. (1-3.)

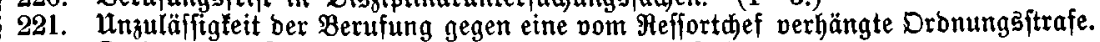

222. Deđtung ber Roften in Disziplinarunterfudungşadjen.

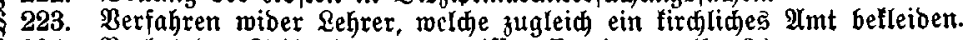

224. Berbot ber Teilnahme an gerviffen Bereinen. (1-3.)

225. Berbot ber Beteiligung an offentfiden Demonftrationen uno $\mathfrak{A}$ gitation gegen bie Regiernng. 


\section{Anttşu[perfion.}

\$ 226. 3ahlung ber Gehaltähälfte an jufpendierte Zefhrer.

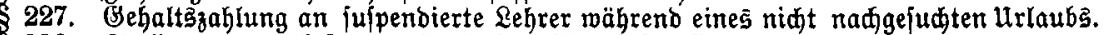
228. Etelloertretungăfoiten während ber $\mathfrak{A}$ mtajufpenjion.

\$229. M̉enn auf ftrafredtlide Freifpredung Entlajing folgt, wirb ber einbehaltene Teil bes (Behalts nidit aus̄gezablt.

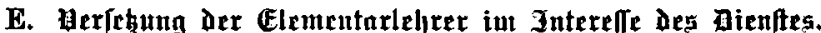

\$230. Bulafjung ber Berfekung in ein anderes $\mathfrak{A}$ mt von niajt geringerem $\mathfrak{A} a n g e . ~(1-2$.

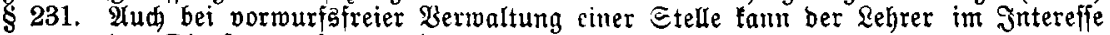
bc马 Dienftes verjetst werten.

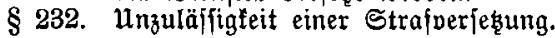

\section{F. fî̄udinumgsfilt.}

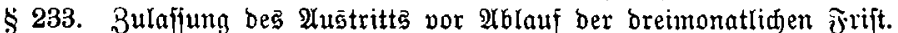

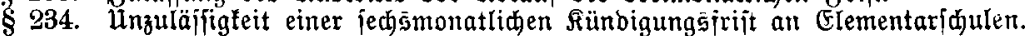

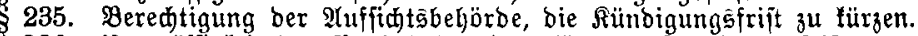

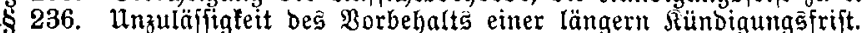

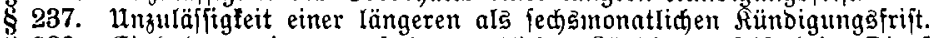

238. Einhaltung einer ev. Fedșmonatliđen Rünbigungş̄rift beim Dienjtaus̆tritt.

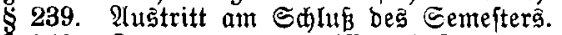

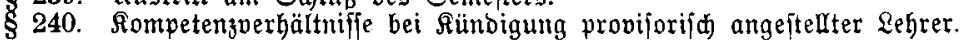

\section{G. Leridjirdenr Angrienenhriten.}

\$241. Inzeige bei Borlabung als Sactuerftänbiger oder Zeuge.

242. Seranjiehung Der Rehrer zur Einquartierungs̆laft.

8243. Zuläffigfeit Der Seranziehung zu ben Einquartierungşlaften.

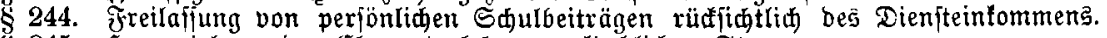

$\$ 245$. Seranziehung Der Elementarlebrer ż firdtiden Steuern.

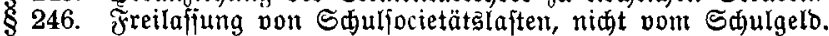

247. Seranziehung ber \&ehrer zur Unterbaltung ber Eocietätslajter. (1-2.)

248. Eine Erhöhung des Rehrereinfommens infolgc Serminberung beăjelben burd Seranziehung zu ben Sdulifteuern ift nid)t zu vermeiben.

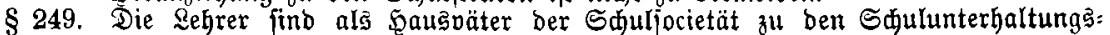
beiträgen heranzugiehen.

\$250. Sđulgelbzahlung §er \&ehrer für eigene Kinder.

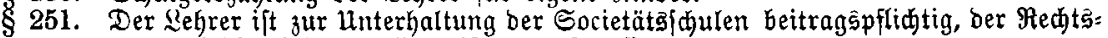
wen auf bie Beitragşpflidt ijt ausgeidloffen.

\$252. Freilaflung Der Elementarlehrer von bireften Fommunalauflagen.

\$253. ¿ehrer von Mittel= unb höheren ober mittleren Mäbdenidulen find fommunal= jteuerpflichtig.

\$ 254. Berfauf von Sdireibmaterialien feiteng ber Rehrer. (1-2.)

255. Berfauf von Shulbüdern burd bie Rehrer.

256. Sinjidttlid ber jtaaţbürgerliden Hedte und \$flidjten nimmt ber Rehrer feine Âus̄nahmejtellung ein.

§ 257. Die 3ulaffung Der Ėlementarlebrer zur \$rüjurg pro facultate docendi ift auggejकlofien. (1-2.)

\$258. Berleihung von Titeln.

259. Maximalzahl ber Bffiditumben.

260. Beteiligung ber Rehrer bet Rirdenvifitationen.

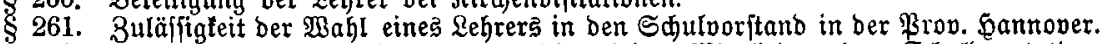

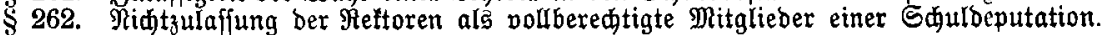

263. 3uziehung bes Meftors zu ben Siłungen ber Sduldeputation.

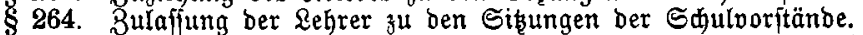

\$ 265. Ünzuläffigteit ber Berpflidtung Der Lehrer, Den Sindern anderer Konfeffion ben Religionsunterridt zu erteilen.

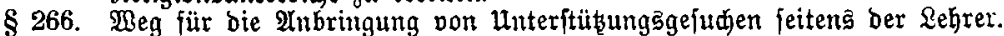

267. 2unnahme eines \$räparanden zur Đilfe beš ¿ehrers.

268. Bermietung eines Teils ber Qehrerwohnung.

3269. 3ur Bermietung ber Dienftwohnung bebarf es ber Buftimmung Der Bemeinbe, fowie ber Genehmigung ber Sdulauffidhtabehörbe.

\$270. Urlaubsnadfudjungen für Qehrer.

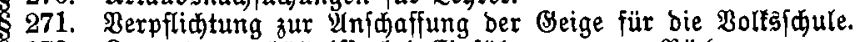

272. Fompetenzyerhältnifje bei Einführung neuer Büdjer.

273. Einführung neuer \&ehrbüder.

§ 274. Bereibigung bes \&ehrers. 


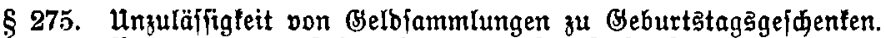

276. Benukung von Sdullofalen zum İmpfgejdäft.

277. Unbefugtes Betreten bes Sdjulzimmers.

278. Eingriffe in bie Disjiplin.

279. Rompetenz ber \&anbräte in Bezug auf bie Rehrer.

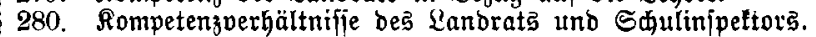

281. Gegen bie Rehrer tann nur bie Reqierung Dronungştrafe verbängen.

282. Folleftiveingaben von $\mathfrak{E}$ hrerinnen find nidt geftattet.

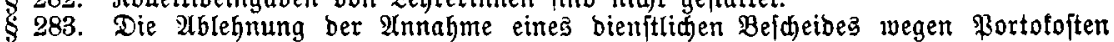
ift nid)t zuläffíg. $(1-2$.

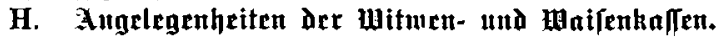

\$284. Seie nom 22. Dezember 1869.

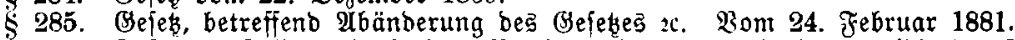

\$286. Bujammenftellung berjenigen \$unfte, über welde in ben revibierten Statuten ber Qehrerwittmen = und פaifenfaffe Beftimmungen zu treffen find.

\$ 287. Bon eimer Rehreritelle, weldhe befintitiv mit einer \&ehrerin befegt wirb, find Witmentaffertbeiträge nidt zu erhebert.

§ 288. Witwenpenjion wiro nidt gewährt, wenn eine \$itwe bemnädft mit einem nidj bem Rehreritanbe angehörigen Manne verbeiratet geweien ift.

§ 289. Beredtigung ber provijorij内 angeftellten Refrer auf bie Mitgliebidaft bei ben Qehrerwitwen = und Maifenfafien.

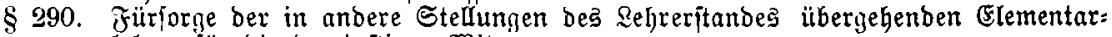
lehrer für bie bereinftiten $\mathfrak{B}$ itmen.

\$291. Die Ruratoren Der \&ehrenvitwentaffen find in alfen Das Raffenvermögen be: treffenden Fra!̣en zu Gören.

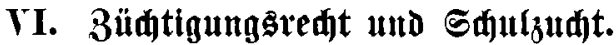

\section{A. Jü̈thtigunngredjt.}

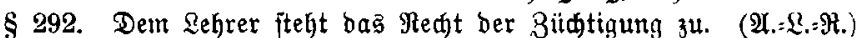

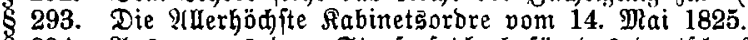

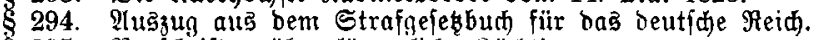

295 . Boridriften über förperlide 及ïdtigunt?.

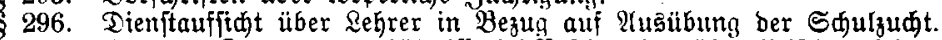

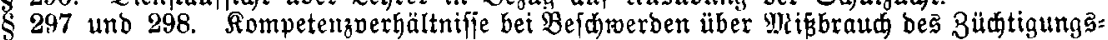
rectits. $(1-9$.

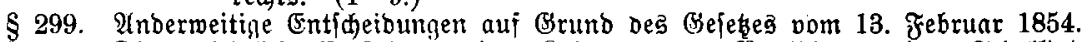

299a. Die geridtlide Nerfolgung eines Rehrers weglen Beleibigung eines Sdultinbes

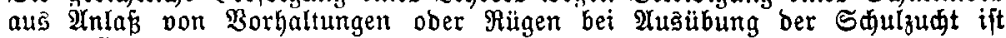
unz̧uäffịg. (1-2.)

B. Sdjulyudt ithrrbaupt.

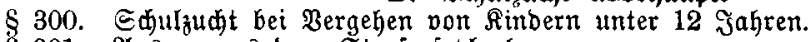

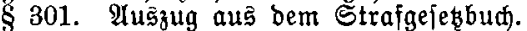

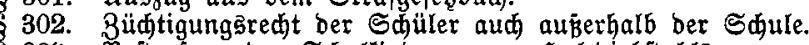

303. Beftrafung Der Sdulfinoer wegen Solzbiebftabls.

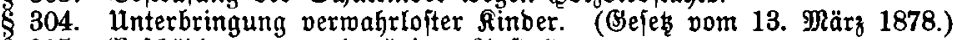

305. Beiđäbigung gemeinnüłiger 2injtalten.

\$306. Berrenbung ber Rinber öu öffentliden Sdauftellungen.

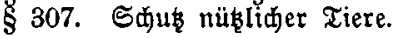

§308. Heję pom 11. Diärz 1872.

\section{Sđulauffidnt.}

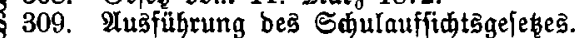

310. Meitere Ausführung beşfelben.

\$10a. Bei Bewerbungen find leine Beugniffe auşzuftellen.

\section{Yehörden und Drgane der Sđaulaufint.}

\section{A. Bir Riegterung.}

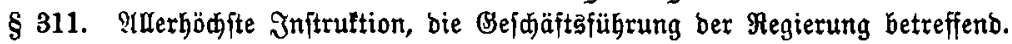

B. Ber fireis/dulinfpektor.

$\S 312$ unb 313. Эnitruftion. - Medte. - Pflidten $2 c$.

C. fokalldulinfprktor.

\$314. \$fliditen ber Sdulauffeher.

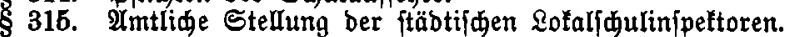

316. Bertretung beurlaubter \$rebiger in ber Sdulinfpettion. 


\section{Squuldeputativuru.}

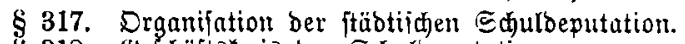

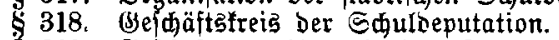

319. Gefdäftsverteilung in ben Sdulbeputationen.

320. SteIlung ber ftäbtifhen Sđulbeputation zu Etaat unb Genteinbe.

321. Stellung bes şürgermeifters̄ zur ftäbtijめen Sdulbeputation.

\$ 321a. Befud ber Édule feiteng einzelner Mitglieber ber Sđulbeputation.

\section{E. Bar shuluorttatid.}

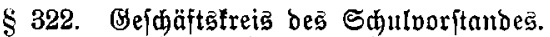

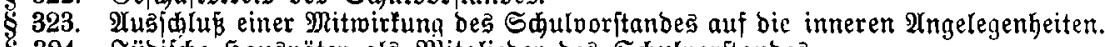

324. Jübifhe Sausväter als Mitglieber bes Equlvorftanbes.

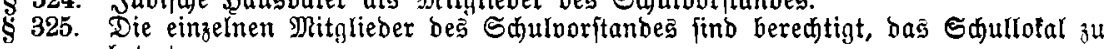
betreten.

\section{F. Stndtuerordurteunerfanmmlıng.}

$\S 326$. Stellung ber Stabtveroronetenveriammlung zu ben ftäbtifden Sđulangelegen: heiten. (1-2).

\section{G. Bir Pandrätr.}

\$327. Beteiligung ber \&anbräte an ben Sdulrevifionen. (1-2.)

\section{H. fjaupflabrer uth Kaktoren.}

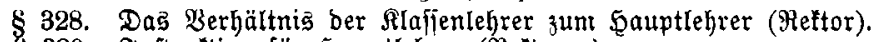

329. §nftruftion für Б口auptlefrer (Reftoren).

\$3 330. Stumbenzahl jür Rettoren.

\section{Tie @đulbcrmaltuty. \\ A. Bir Brefundlyritapflegr.}

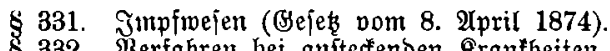

332. Berfahren bet antecfenden Rramtheiten.

333. Desinfettion.

§33a. (Sefunbhetispflege in ber Bolfajdule.

\section{B. Gd)ulzูwnun.}

$\$ 334$. Borjüriften beș altgemeinen Ranbredts.

335. 2Ullerfödite Г̃abinetsorore vom 14. Mai 1825 .

336. 3mangsweifes beftellen zut Shule.

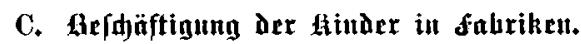

§ 337. 2łbänberung ber (Sewerbeorbungägefeţe vom 17. Juli 1878.

\section{Bas fjütruelert.}

338. Bebingungen für bie Erteilunģ von Sütejdeinett.

339. Squlunterridt Der Sütefinder. $(1-3)$.

340 . Befłulung ber šütefinder.

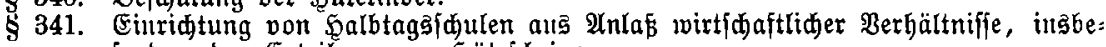
jonbere ber Erteilung von Sütejळeinen.

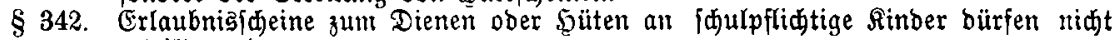
erteilt werber.

\section{Antıng.}

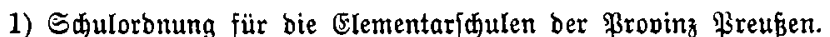

2) Shulteglement für bie nieberen fatholijøen Sđulen in ben Stäbten und auf

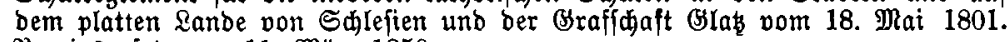

3) Bereingageje pom 11. Miärz 1850 .

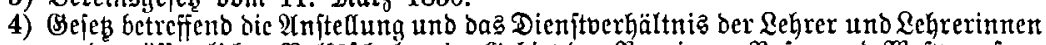

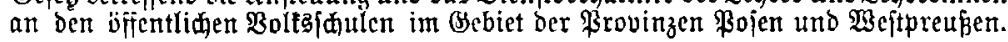

